\title{
ACRITARCAS SILURIANOS DA FORMAÇÃO TROMBETAS, BACIA DO AMAZONAS
}

\author{
Sandra de Fátima OLIVEIRA \\ Murilo Rodolfo de LIMA
}

\section{RESUMO}

A análise palinológica de uma amostra procedente da cachoeira do Viramundo, parte inferior do Membro Pitinga, Formação Trombetas, revelou presença de uma palinoflora diversificada e bem preservada. Vinte e três espécies de acritarcas foram identificadas, incluindo algumas com valor estratigráfico, indicativas do intervalo bioestratigráfico correspondente ao Ludloviano. Esta atribuição reforça conclusões obtidas pelos últimos autores que trabalharam na área, apoiando a idéia de contato gradacional entre a Formação Trombetas e a sobrejacente Formação Maecuru.

A assembléia foi certamente depositada em um ambiente marinho, nerítico e provavelmente sob condições de salinidade baixa.

\section{ABSTRACT}

Analysis of a sample from the Viramundo falls, lower part of the Pitinga Member, Trombetas Formation, reveals a diversified and well-preserved palynoflora. Twenty-three species of acritarchs are identified, including several guide-fossils, indicative of a biostratigraphic interval corresponding to the Ludlovian. This assignment reinforces recent conclusions by other authors, supporting the idea of a gradational contact between the Trombetas Formation and the overlying Maecuru Formation.

The assemblage was certainly deposited in a neritic marine environment, probably under low salinity conditions.

\section{INTRODUÇÃO}

A Cachoeira do Viramundo representa hoje uma localidade clássica de ocorrência de sedimentos da Formação Trombetas. Conhecida desde o século passado, sua seqüência sedimentar foi, junto com a da cachoeira Porteira, utilizada por DERBY (1878) para conceituação do então chamado "grés de Trombetas". Situa-se no baixo curso do rio Trombetas, cerca de $4 \mathrm{~km}$ antes do seu encontro com o rio Mapuena (Fig. 1).

Ricamente fossilíferos, os níveis estratigráficos referidos notabilizam-se principalmente pela ocorrência de graptozoários ainda mal estudados, entre os quais se sobressai, pela abundância, a espécie Climacograptus innotatus NICHOLSON, var. brasiliensis RUEDEMANN. A datação desta espécie, efetuada por MAURY (1929), tem mantido para a Formação Trombetas uma Idade Siluriana Inferior. Esta, contudo, encontra-se cada vez mais em desacordo com a atribuída por outros fósseis presentes. $\mathrm{O}$ objetivo deste trabalho é analisar a informação concernente aos acritarcas associados aos níveis onde ocorre este graptozoário, pondo em discussão a validade de tal atribuição cronológica.

\subsection{Estratigrafia}

De acordo com o ponto de vista mais aceito pelos autores, expresso na literatura consultada, a Formação Trombetas ocorre nas bacias do Amazonas e Solimões. Segundo SANTOS (1984), aflora nos flancos nonte e sul, em faixas estreitas compreendidas entre o alto de Gurupá e Purus. Na porção central da bacia (Médio Amazonas) a espessura é da ordem de $1.000 \mathrm{~m}$. De acordo igualmente com a literatura consultada, a unidade é representada por quatro membros. São eles, da base para o topo: Membros Autaz-Mirim, Nhamundá, Pitinga e Manacapuru.

O membro Autaz-Mirim é essencialmente psamítico, sendo composto por arenitos vermelhos basais, que passam a brancos no topo da seção. A espessura máxima é estimada em $290 \mathrm{~m}$. Ocorrem nesta seqüência importantes intercalações de folhelhos sílticos cinza-esverdeados. A estratificação predominante é plano-horizontal, ocorrendo bioturbações verticais.

O Membro Nhamundá é composto por quartzo-arenitos representativos de ambiente nerítico, geralmente de cor branca. Ocorrem in- 


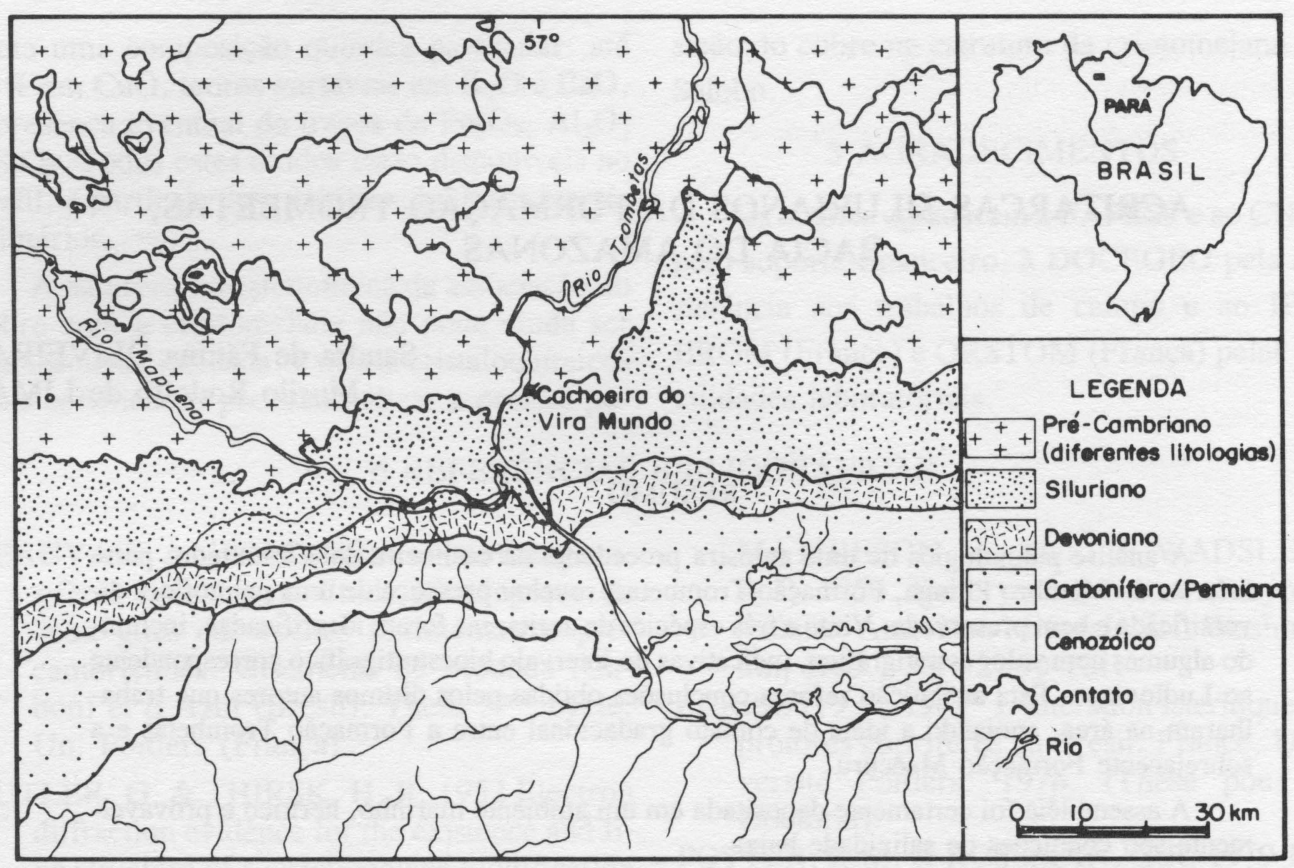

FIGURA 1 - Mapa de localização do afloramento (adaptado do projeto RadamBrasil, 1976).

tercalações de folhelho cinza no topo, sendo a espessura estimada em cerca de $340 \mathrm{~m}$.

O Membro Pitinga é a única das quatro unidades presente no vale do rio Trombetas. Conta com litologias variadas, as mais basais aflorando na cachoeira do Viramundo. Correspondem a siltitos, folhelhos e arenitos finos. Acima, a unidade torna-se mais arenítica, passando a exibir estratificação cruzada. A parte mais superior é constituída por siltitos e folhelhos cinzentos, com arenitos e níveis de sílex associados. A espessura máxima é da ordem de $260 \mathrm{~m}$.

O Membro Manacapuru é representado por arenitos com matriz argilosa, acinzentados, bem como por níveis sílticos. É notável a presença de camadas sideríticas na base e no topo deste membro, associadas aos arenitos. A espessura máxima encontrada é da ordem de $220 \mathrm{~m}$.

\subsection{Paleontologia}

A Formação Trombetas é mais fossilífera na sua parte superior. O Membro Autaz-Mirim é o mais pobre de toda a unidade. Segundo a literatura, ocorrem quitinozoários raros e mal preservados, graptozoários e espículas de esponjas. Também pobre, o Membro Nhamundá apresenta, em algumas camadas de folhelhos, raros quitinozoários, acritarcas e esporomorfos indeterminados. Em leitos de sílex ocorrem espículas de esponjas. Em sondagem foi constatada a presença de braquiópodes, cifozoários e cefalópodes (QUADROS, 1985). O mais rico de toda a seção é o Membro Pitinga. Na parte basal (sopé da cachoeira do Viramundo) ocorre grande quantidade de graptozoários associados a siltitos, folhelhos e arenitos finos. Destas camadas foi também descrita importante associação de quitinozoários (COSTA, 1972). Alguns metros acima, níveis areníticos portam notável fauna descrita por CLARKE (1899), composta por braquiópodes, bivalves, gastrópodes, cefalópodes, tentaculitídeos, ostracodes e cifozoários. Ainda mais acima começam a ocorrer abundantes traços fósseis. Na parte superior do membro foi recentemente referida por JANVIER \& MELO (1988) importante associação de peixes placodermes (acantódeos). Em sondagem foram encontrados todos os grupos mencionados além de acritarcas, escolecodontes, foraminíferos arenáceos, espículas de esponjas e esporomorfos. Finalmente, de acordo com a literatura consultada, o Membro Manacapuru apresenta abundantes graptozoários, esporomorfos, quitinozoários, espículas de esponjas e acritarcas.

\subsection{Dados palinológicos}

\subsubsection{Dados prévios}

DAEMON \& CONTREIRAS (1971) efetuaram zoneamento palinológico da Bacia do Amazonas. Neste trabalho, os autores incluíram dados de sondagem relativos à Formação Trombetas. Com base na presença de espécies de acri- 
tarcas, DAEMON \& CONTREIRAS (op.cit.) admitiram Idade Llandloveriana para a unidade. Conforme pode ser observado, o nível de abordagem sistemática deste trabalho não permite um maior grau de segurança na atribuição efetuada.

QUADROS (1985), em trabalho sobre a Bacia do Amazonas, identificou também formas de acritarcas do Membro Pitinga. A partir da ocorrência de nove espécies presentes em testemunhos de sondagem, o autor atribuiu Idade Gediniana aos níveis portadores.

\subsubsection{Proveniência do material estudado}

A amostra estudada faz parte do acervo do IG/USP. Corresponde a um arenito castanho, silicificado, contendo abundantes restos de Climacograptus innotatus NICHOLSON var. brasiliensis RUEDEMANN. Foi coletada no sopé da cachoeira do Viramundo representando os níveis mais basais do Membro Pitinga. Foi processada segundo téćnica palinológica padrão. A partir de resíduo obtido foram montadas 6 lâminas, que se encontram na coleção de entrada do DPE/IG-USP sob os números GP/4E-1317 a GP/4E-1322.

\subsubsection{Resultados obtidos}

Vinte e três espécies de acritarcas foram reconhecidas. O predomínio absoluto é de espécies pertencentes aos gêneros Multiplicisphaeridium e Veryhachium.

Para identificação das espécies foram consultados, entre outros, os trabalhos de BRITO (1967), BRITO \& SANTOS (1965), COMBAZ et al. (1967), CRAMER (1964, 1966, 1968, 1971), DEFLANDRE (1956), DORNING (1981), DOWNIE (1960), DOWNIE \& SARJEANT (1963), EISENACK $(1955,1958)$, JARDINÉ et al. (1974), LISTER (1970), PLAYFORD (1977), STAPLIN et al. (1965) e THUSU (1973). A relação total das formas identificadas é a seguinte:

Gênero Baltisphaeridium (EISENACK, 1958) emend. STAPLIN, JANSONIUS \& POCOCK, 1965

Baltisphaeridium gueltaense JARDINÉ, COMBAZ, MAGLOIRE, PENIGUEL \& VACHEY, 1974

(Est. I, fig. 1)

Gênero Dactylofusa (BRITO \& SANTOS, 1965) emend. COMBAZ, LANGE \& PANSART, 1967

Dactylofusa maranhensis (BRITO \& SANTOS, 1965) emend. COMBAZ, LANGE \& PANSART, 1967

(Est. I, fig. 2)
Gênero Diexallophasis LOEBLICH, 1970

Diexallophasis remota (DEUNFF) PLAYFORD, 1977

(Est. I, fig. 3)

Gênero Dictyotidium EISENACK, 1955

Dictyotidium dictyotum (EISENACK) EISENACK, 1955

(Est. I, fig. 4)

Gênero Domasia DOWNIE, 1960

Domasia elongata DOWNIE, 1960

Gênero Estiastra EISENACK, 1959

(Est. I, fig. 5)

Estiastra stellata LOEBLICH, 1970

(Est. I, fig. 6)

Estiastra sp.

(Est. I, fig. 7)

Gênero Eupoikilofusa CRAMER, 1970

Eupoikilofusa striatifera (CRAMER), CRAMER, 1971

(Est. I, Fig. 8)

Gênero Gorgonisphaeridium STAPLIN, JANSONIUS \& POCOCK, 1965

Gorgonisphaeridium bringewoodensis DORNING, 1981

(Est. I, fig. 9)

Gênero Leiofusa EISENACK, 1938

Leiofusa estrecha CRAMER, 1964

(Est. I, fig. 10)

Gênero Leiosphaeridia EISENACK, 1958

Leiosphaeridia $\mathrm{sp}$.

(Est. I, fig. 11)

Gênero Micrhystridium DEFLANDRE, 1937

Micrhystridium stellatum DEFLANDRE, 1946

( Est. I, fig. 12)

Gênero Multiplicisphaeridium (STAPLIN) STAPLIN, JANSONIUS \& POCOCK, 1965 Multiplicisphaeridium ramusculosum (DEFLANDRE) LISTER, 1970

(Est. I, fig. 13)

Multiplicisphaeridium fisheri (CRAMER) LOEBLICH, 1970

(Est. I, fig. 14)

Multiplicisphaeridium sp.

(Est. I, fig. 15)

Gênero Neoveryhachium CRAMER, 1971

Neoveryhachium carminae (CRAMER) CRAMER, 1971

(Est. I, fig. 16)

Gênero Ozotobrachion LOEBLICH \& DRUGG, 1968

Ozotobrachion furcillatus (DEUNFF) PLAYFORD, 1977

( Est. I, fig. 17)

Gênero Tunisphaeridium DEUNFF \& EVITT, 1968

Tunisphaeridium tentaculiferum (MARTIN) CRAMER, 1971

(Est. I, fig. 18) 


\section{Gênero Veryhachium (DEUNFF) DOWNIE \& SARJEANT, 1963 \\ Veryhachium centrigerum DEUNFF, 1957}

(Est. I, fig. 19)

Veryhachium lairdii (DEFLANDRE) exDEUNFF, 1959

(Est. I, fig. 20)

Veryhachium trapezionarion LOEBLICH, 1970

(Est. I, figs. 21 e 22)

Veryhachium trispinosum (EISENACK) DEUNFF, 1954

(Est. I, fig. 23)

Veryhachium wenlockium (DOWNIE) DOWNIE \& SARJEANT, 1963

(Est. I, fig. 24)

\section{DISCUSSÃO E CONCLUSÕES}

\subsection{Idade}

A idade é um dos aspectos mais controvertidos no que se refere ao conhecimento geológico da Formação Trombetas. Desde os trabalhos pioneiros efetuados no século passado (DERBY, 1878; CLARKE, 1899) uma Idade Ordoviciana Superior/Siluriana Inferior foi sugerida para estratos hoje interpretados como pertencentes ao Membro Pitinga. No decorrer deste século, outros trabalhos (KATZER, 1903; SCHUCHERT, 1906; MAURY, 1929) reforçaram esta idéia, fixando a idade no Llandloveriano pela ocorrência de Climacograptus innotatus NICHOLSON var. brasiliensis RUEDEMAN, Orthis callactis DALMANN e do icnofóssil Arthrophycus harlani CONRAD. Tal idade chegou a influenciar trabalhos relativamente modernos, como o de CAPUTO et al. (1972), que posicionaram os membros mais basais da unidade (Membros Autaz-Mirim e Nhamundá) no Ordoviciano, por estarem sotopostos ao Membro Pitinga. COSTA (1972), ao descrever associação de quitinozoários do Membro Pitinga, também atribuiu Idade Llandloveriana para os níveis estudados.

LUDWIG (1964) foi o primeiro autor a discordar deste posicionamento, admitindo, com ba- ses sedimentológicas, um contato gradacional entre as Formações Trombetas e Maecuru, esta de Idade Devoniana Inferior. QUADROS (1985) ao admitir Idade Ludloviana/Gediniana para os Membros Pitinga e Manacapuru, com base em acritarcas e quitinozoários - de certa forma reforçou a idéia de LUDWIG (op. cit.). Esta, entretanto, encontrou oposição de vários autores que se têm dedicado ao tema, exatamente pela forte influência da datação obtida através dos graptozoários e braquiópodes. Mais recentemente, JANVIER \& MELO (1988) retomaram o assunto através do estudo de espinhos, escamas e dentes de peixes acantódeos, presentes na parte superior do Membro Pitinga. Segundo os autores, os restos de vertebrados encontrados são similares aos que ocorrem na Formação Catavi (Bolívia), de Idade Devoniana Inferior, e não podem ser mais antigos que o Siluriano Terminal. Este ponto de vista volta a contestar a idade tradicionalmente atribuída. JANVIER \& MELO (op. cit.) efetuaram ainda uma análise dos fósseis mais importantes, mencionando o fato de o gênero Climacograptus ocorrer até no Devoniano Inferior da Europa e de o táxon Orthis callactis ser, na verdade, um dalmanelídeo indeterminável. Citam ainda análises palinológicas inéditas efetuadas por J. GRAY, mostrando que as assembléias palinológicas do Membro Pitinga sugerem Idade Pós-Llandloveriana.

Os resultados aqui divulgados, os primeiros obtidos a partir de afloramento da unidade, tendem a confirmar estas idéias. Entre os táxons identificados ocorrem várias espécies de valor estratigráfico (Fig. 2), permitindo a atribuição de Idade Ludloviana para o nível portador. Esta datação reforça também o conceito preconizado de contato gradacional entre o Membro Manacapuru (situado acima) e os sedimentos da Formação Maecuru.

\subsection{Ambiente}

A associação estudada, indubitavelmente marinha, é sugestiva de deposição em ambiente nerítico. 


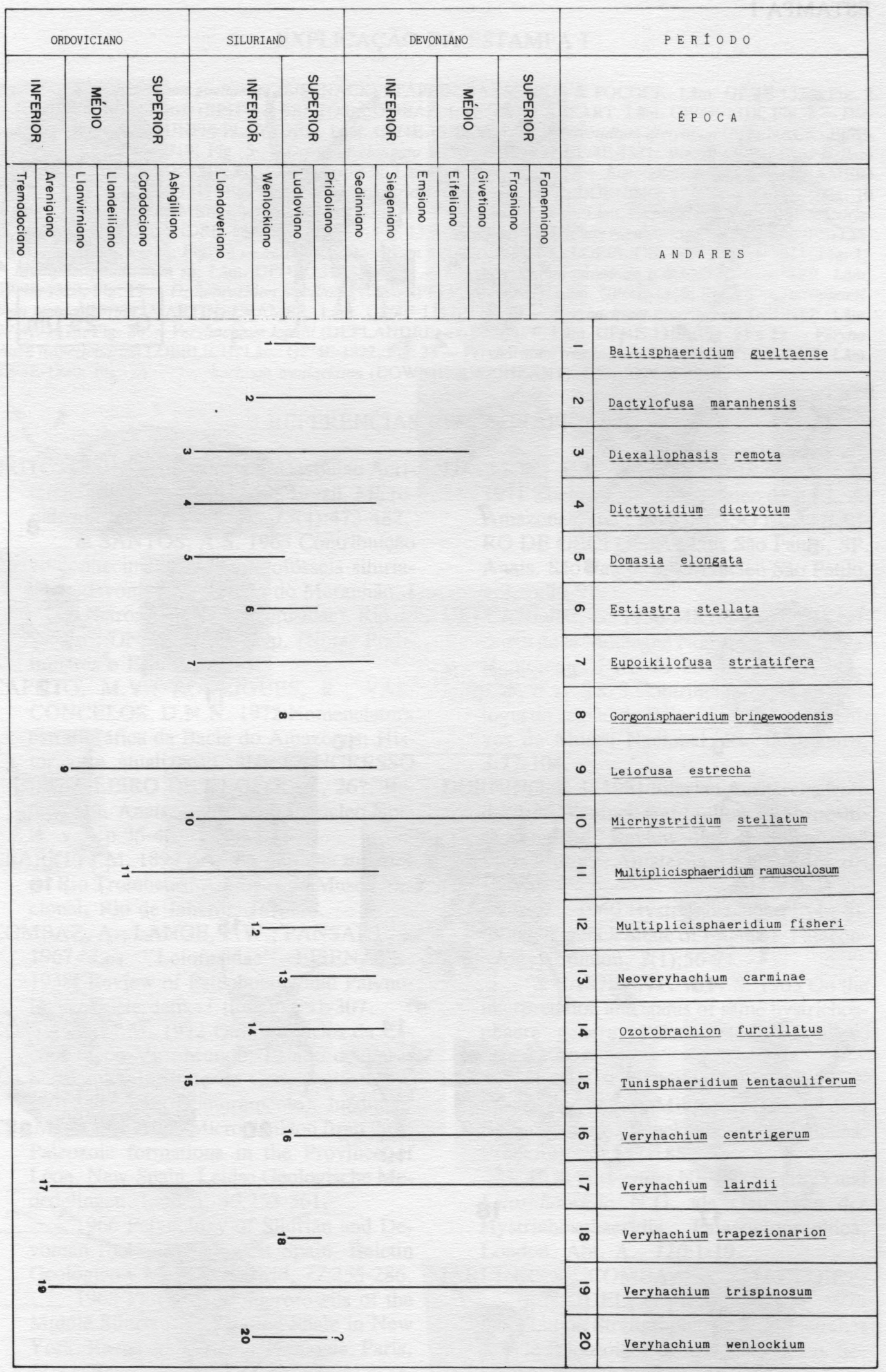

FIGURA 2 - Distribuição cronológica das espécies identificadas (baseada em LISTER, 1970; LOEBLICH, 1970; THUSU, 1973; JARDINÉ et al., 1974 e DORNING, 1981). 


\section{ESTAMPA I}

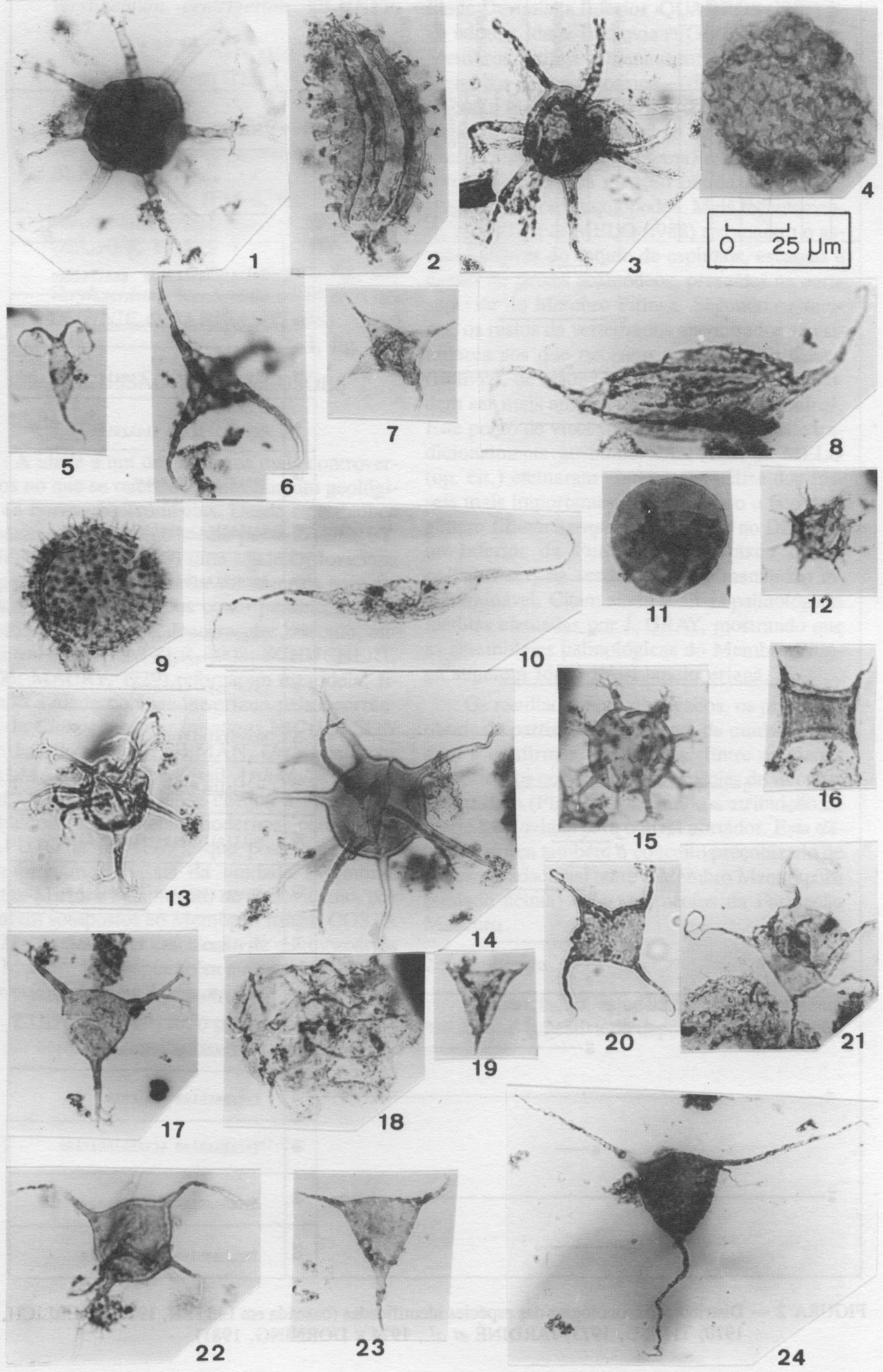




\section{EXPLICAÇÃO DA ESTAMPA I}

Fig. 1 - Baltisphaeridium gueltaense (EISENACK) STAPLIN, JANSONIUS \& POCOCK. Lâm. GP/4E-1322; Fig. 2 - Dactylofusa maranhensis (BRITO \& SANTOS) COMBAZ, LANGE \& PANSART. Lâm. GP/4E-1318; Fig. 3 - Diexallophasis remota (DEUNFF) PLAYFORD. Lâm. GP/4E-1318; Fig. 4 - Dictyotidium dictyotum (EISENACK) EISENACK. Lâm. GP/4E-1319; Fig. 5 - Domasia elongata DOWNIE. Lâm. GP/4E-1321; Fig. 6 - Estiastra stellata LOEBLICH. Lâm. GP/4E-1319; Fig. 7 - Estiastra sp. Lâm. GP/4E-1321; Fig. 8 - Eupoikilofusa striatifera (CRAMER) CRAMER. Lâm. GP/4E-1317; Fig. 9-Gorgonisphaeridium bringewoodensis DORNING, Lâm. GP/4E-1317; Fig. 10 - Leiofusa estrecha CRAMER. Lâm. GP/4E-1321; Fig. 11 - Leiosphaeridia sp. Lâm. GP/4E-1317; Fig. 12 - Micrhystridium stellatum DEFLANDRE. Lâm. GP/4E-1320; Fig. 13 - Multiplicisphaeridium ramusculosum (DEFLANDRE) LISTER. Lâm. GP/4E-1318; Fig. 14 - Multiplicisphaeridium fisheri (CRAMER) LOEBLICH. Lâm. GP/4E-1321; Fig. 15 - Multiplicisphaeridium sp. Lâm. GP/4E-1317; Fig. 16 - Neoveryhachium carminae (CRAMER) CRAMER. Lâm. GP/4E-1320; Fig. 17 - Ozotobrachion furcillatus (DEUNFF) PLAYFORD. Lâm. GP/4E-1319; Fig. 18 - Tunisphaeridium tentaculiferum (MARTIN) CRAMER. Lâm. GP/4E-1317; Fig. 19 - Veryhachium centrigerum DEUNFF. Lâm. GP/4E-1318; Fig. 20 - Veryhachium lairdii (DEFLANDRE) ex-DEUNFF. Lâm. GP/4E-1319; Fig. 21 e 22 - Veryhachium trapezionarion LOEBLICH. Lâm. GP/4E-1322; Fig. 23 - Veryhachium trispinosum (EISENACK) DEUNFF. Lâm. GP/4E-1320; Fig. 24 - Veryhachium wenlockium (DOWNIE \& SARJEANT). Lâm. GP/4E-1319.

\section{REFERÊNCIAS BIBLIOGRÁFICAS}

BRITO, I.M. 1967 Silurian and Devonian Acritarcha from Maranhão Basin, Brazil. Micropaleontology, New York, 13(4):473-482.

\& SANTOS, A.S. 1965 Contribuição ao conhecimento dos microfósseis silurianos e devonianos da Bacia do Maranhão. I - Os Netromorphitae (Leiofusidae). Rio de Janeiro, DNPM/DGM. 29p. (Notas Preliminares e Estudos, 129).

CAPUTO, M.V.; RODRIGUES, R.; VASCONCELOS, D.N.N. 1972 Nomenclatura estratigráfica da Bacia do Amazonas; Histórico e atualização. IN: CONGRESSO BRASILEIRO DE GEOLOGIA, 26\%, Belém, PA. Anais. Belém, SBG/Núcleo Norte. v.3. p.35-46.

CLARKE, J.M. 1899 A fauna siluriana superior do Rio Trombetas. Archivos do Museu Nacional, Rio de Janeiro, 10:1-48.

COMBAZ, A.; LANGE, F.W.; PANSART, J. 1967 Les "Leiofusidae" EISENACK, 1938. Review of Paleobotany and Palynology, Amsterdam, 1 (único):291-307.

COSTA, N.M.M. 1972 Quitinozoários da Cachoeira do Vira-Mundo, Estado do Pará. São Paulo, Instituto de Geociências/USP. 167p. (Tese de Doutoramento). Inédita.

CRAMER, F.H. 1964 Microplankton from three Paleozoic formations in the Province of Leon, New-Spain. Leidse Geologische Mededelingen, Leiden, 30:253-361.

1966 Palynology of Silurian and Devonian rocks in northwest Spain. Boletin Geológico y Minero, Madrid, 77:255-286.

1968 Palynologic microfossils of the Middle Silurian Maplewood Shale in New York. Revue de Micropaléontologie, Paris, 11:61-70.

1971 Distribution of selected Silurian acritarchs. Revista Española de Micropaleontologia, Madrid, (núm. extr.). 202p.
DAEMON, R.F. \& CONTREIRAS, C.J.A. 1971 Zoneamento palinológico da Bacia do Amazonas. IN: CONGRESSO BRASILEIRO DE GEOLOGIA, 25․, São Paulo, SP. Anais. São Paulo, SBG/Núcleo São Paulo. v.3. p.79-91.

DEFLANDRE, G. 1956 Microfossiles des calcaires de la Montagne Noire. Annales de Paléontologie, Paris 31:39-75.

DERBY, O.A. 1878 Contribuições para a geologia da região do Baixo Amazonas. Archivos do Museu Nacional, Rio de Janeiro, 3:77-104.

DORNING, K.J. 1981 Silurian Acritarchs from the type Wenlock and Ludlow of Shropshire, England. Review of Palaebotany and Palynology, Amsterdam, 34 (único): 175-203.

DOWNIE, C. 1960 Hystrichospheres from Silurian Wenlock Shale of England. Palaeontology, London, 2(1):56-71.

\& SARJEANT. W.A.S. 1963 On the interpretation and status of same hystrichosphaere genera. Palaeontology, London, 6(1):83-96.

EISENACK, A. 1955 Chitinozoen, Hystrichosphären und andere Mikrofossilien aus dem Beyrichia Kalk. Senckenbergiana Lethaea, Frankfurt, 36:157-188.

1958 Tasmanites NEWTON, 1875 und Leiosphaeridia N.G. als Gattungen der Hystrichosphaeridia. Palaeontographica, London, Abt. A., 110:1-19.

JARDINÉ. S.; COMBAZ, A.; MAGLOIRE, L.; PENIGUEL, G.; VACHEY, G. 1974 Distribution Stratigraphique des acritarches dans le Paleozoique du Sahara Algérien. Review of Palaeobotany and Palynology, Amsterdam, 18 (único):99-129.

JANVIER, P. \& MELO, J.H.G. 1988 Acanthodian fish remains from the Upper Silurian 
or Lower Devonian of the Amazon Basin, Brazil. Palaeontology, London, 31(3): 771-777.

KATZER, F. 1903 Grundzüge der Geologie des unteren Amazonas-gelreter (des Staates Para in Brasilien). Leipzig, 1903. 296p.

LISTER, T.R. 1970 A monograph of acritarchs and Chitinozoa from Wenlock and Ludlow series of the Ludlow and Millichope areas, Shropshire, part 1. London, Palaeontographical Society. 100p. (Monography, 124).

LOEBLICH JR., A.R. 1970 Morphology, ultrastruture and distribution of Paleozoic acritarchs. IN: PROCEEDINGS NORTH AMERICAN PALEONTOLOGY CONVENTION, Chicago, 1969. G:705-788.

LUDWIG, G. 1964 Nova divisão estratigráfica e correlação faciológica por meio de pequenas estruturas internas dos sedimentos silurianos e devonianos da Bacia do Médio Amazonas. Ciência-Técnica-Petróleo, Rio de Janeiro, 1:1-72.

MAURY, C.J. 1929 Uma zona de graptolitos do Llandovery Inferior no Rio Trombetas, Estado do Pará, Brasil. Rio de Janeiro, DNPM/SGM. 53p. (Monografia, 7)

PLAYFORD, G. 1977 Lower to Middle Devonian acritarchs of Moose River Basin,
Ontário. Ottawa, Geological Survey of Canada. 87p. (Bulletin, 279).

QUADROS, L.P. 1985 Distribuição Bioestratigráfica dos Chitinozoa e Acritarchae na $\mathrm{Ba}$ cia do Amazonas. Rio de Janeiro, Instituto de Geociências/UFRJ. 179p. (Tese de Doutoramento). Inédita.

RADAMBRASIL. 1976 Departamento Nacional da Produção Mineral, Levantamento de Recursos Naturais, vol.10, Folha SA. 21 Santarém, 522A, 7 mapas.

SANTOS, J.O.S. 1984 A parte setentrional do cráton Amazônico (Escudo das Guianas) e a Bacia Amazônica. IN: SCHOBBENHAUS, C.; CAMPOS, D.A.; DERZE, G.R.; ASMUS, H.E. (Coords.). Geologia do Brasil. Brasília, DNPM. p.57-91.

SCHUCHERT, C. 1906 Geology of the Lower Amazon region. Journal of Geology, Chicago, 14(8):722-746.

STAPLIN, F.L.; JANSONIUS, J.; POCOCK, S.A.J. 1965 Evolution of some acritarchous hystrichosphere genera. Neues Jahrbuch Geologie Palaeontologie. Abh, 123:167-201.

THUSU, B. 1973 Acritarchs of the Middle Silurian Rochester Formation of Ontario. Palaeontology, London, 16(4):799-826.

Manuscrito recebido em setembro de 89

Endereço dos autores:

- Sandra de Fátima Oliveira - (Pós-graduanda do IG/USP e Bolsista da FAPESP - Proc. 89/1799-1) - Universidade de São Paulo - Instituto de Geociências - Caixa Postal 20.899 - 01498 - São Paulo, SP - Brasil.

- Murilo Rodolfo de Lima - Universidade de São Paulo - Instituto de Geociências - Caixa Postal 20.899 - 01498

- São Paulo, SP - Brasil. 